\title{
A Range-Based Three Dimensional Node Self-Localization Algorithm for Wireless Sensor Network
}

\author{
Yanhui Lv, Sitong Meng, Deyu Zhang and Yunfei Huang \\ College of Information Science and Engineering, Shenyang Ligong University \\ yanhuilv@126.com
}

\begin{abstract}
The node localization technology is one of the key technologies in wireless sensor network. Considering the following problems that most of existing localization algorithms are based on the two dimensional environment, and the error in the multi-hop ranging is easy to accumulate, a range-based three dimensional nodes self-localization algorithm is presented in this paper. First, based on the topological relation between the anchor nodes and the unknown nodes in the three dimensions, the basic principle and calculation process of the node self-localization algorithm are described. On this basis, the procedure of the localization algorithm is given. In addition, the two-hop ranging method is introduced, which can effectively reduce the error accumulation caused in the calculation of the node coordinates. The simulation results indicate that the proposed algorithm has higher localization precision and localization coverage rate compared with the traditional quadrilateral localization algorithm.
\end{abstract}

Keywords: Range-based, Localization, Localization precision, Localization coverage rate, Wireless sensor network

\section{Introduction}

The wireless sensor network, which is composed of plenty of static or mobile sensor nodes in a self-organization and multi-hop manner, plays an important role in the military and civilian fields [1-2]. Many applications of wireless sensor network require the node to be able to get its own location information to determine the location of the monitoring events or objectives. Therefore, the node localization technology is the foundation of all kinds of concrete applications of the wireless sensor network [3-4].

Localization of nodes in the wireless sensor network refers to the process in which the sensor nodes can obtain the location information according to the location information of some anchor nodes by a certain localization technology [5]. In many occasions, the sensor nodes are randomly deployed in an area, and they do not know their location information in advance. Therefore, it is needed that each node obtains respective location information by using corresponding localization technology after it is disposed. At present, the commonest localization technology is GPS. Although a node can obtain its location information by equipping itself with the global positioning system (GPS), the limited energy and lower cost of every node make it unrealistic to install GPS system on all the nodes. Therefore, it is necessary to study the localization technologies that are suitable for the wireless sensor network.

In recent decade, many node self-localization algorithms have been proposed in WSN. According to the different localization methods, the localization algorithms can be classified as follows.

The range-based and range-free localization algorithms [6-7]. Comparing with the range-free localization algorithm, the localization precision of the range-based localization algorithm will be higher in the case of higher ranging precision, although the computation and communication of the algorithm are larger [8-9]. The well-known range- 
based localization algorithms include RSSI (Received Signal Strength Indication) [10], Euclidean algorithm, and the quadrilateral localization algorithm. The classical range-free localization algorithms include APIT [11], Ad-Hoc positioning system [12], and DV-Hop [13-14].

The centralized and distributed localization algorithms. The deficiency of centralized location algorithm is that the excessive amount of communication of the central node and the nearby node will lead to excessive energy consumption and make them die earlier. So, the distributed localization algorithms are taken as the mainstream localization algorithms at present [15-18].

Most of the existing localization algorithms are based on the two dimensional environment, however the terrain may be uneven in the place in which the WSN is deployed practically, and in this case, the localization error of the nodes will increase obviously. In addition, all the existing localization algorithms are based on the multi-hop ranging, that is, after a node is positioned through the anchor nodes, it will make its own coordinates participating in the coordinate calculation of the new node, and with the increase of the number of hops, the coordinates error of the new node will increase gradually, which will cause the error accumulation. In view of the above-mentioned problems, a range-based three dimensional node self-localization algorithm is proposed in this paper. By establishing the three dimensional coordinate system without adding new hardware equipment, the distance equation of the two-hop ranging of the nodes is derived, further, the procedure of the node self-localization algorithm is given.

The rest of the paper is organized as follows. Section 2 defines some terms used in this paper. Section 3 introduces the basic principle of the range-based algorithm. Section 4 describes the proposed distributed localization algorithm in detail. Section 5 reports the simulation results. Concluding remarks and future directions are presented in Section 6.

\section{Relevant Definition}

Some of the terms used in this paper are defined as follows.

Anchor node: The node is equipped with GPS or other positioning system. After it is disposed, the node can obtain its own absolute coordinates immediately.

Reference node: The node is not equipped with any positioning system, but it has calculated its location coordinates by using the node localization algorithm.

Unknown node: The node is not equipped with any positioning system and its current location information is still unknown. The node waits for being positioned by the localization algorithm.

Known node: It can be an anchor node or a reference node.

\section{The Principle of the Three Dimensional Self-Localization Algorithm}

Suppose $\left(x_{1}, y_{1}, z_{1}\right),\left(x_{2}, y_{2}, z_{2}\right)$, and $\left(x_{n}, y_{n}, z_{n}\right)$ are the coordinates of $\mathrm{n}$ known nodes respectively, $(x, y, z)$ is the coordinates of an unknown node, $d_{1}, d_{2}, \ldots d_{n}$ are the distance from the unknown node to the $n$ known nodes respectively, then the equation (1) can be obtained using the maximum likelihood estimation method.

$$
\left\{\begin{array}{c}
\left(x-x_{1}\right)^{2}+\left(y-y_{1}\right)^{2}+\left(z-z_{1}\right)^{2}=d_{1}^{2} \\
\vdots \\
\left(x-x_{n}\right)^{2}+\left(y-y_{n}\right)^{2}+\left(z-z_{n}\right)^{2}=d_{n}^{2}
\end{array}\right.
$$

In the equation (1), at least four equations are needed to calculate the values of $x, y$ and $z$. That is to say, in the three dimensional case, it is required that only after the unknown node gets the distance from the four or more than four known nodes to it, it can calculate its own coordinates. 
Figure 1 gives the schematic diagram of the node self-localization algorithm in three dimensional spaces.

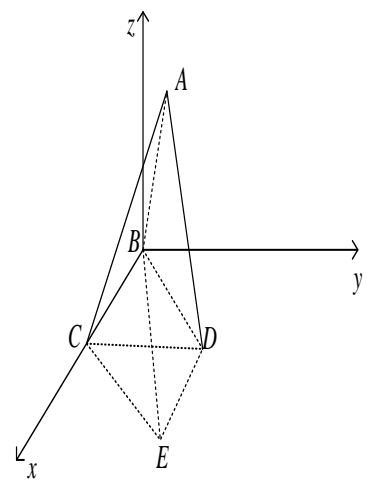

\section{Figure 1. The Schematic Diagram of the Localization Algorithm}

In the Figure 1, suppose that the node $A$ is an anchor node, the node $E$ is an unknown node, the node $A$ is the neighbor node of the node $B, C$ and $D$, the node $E$ is the neighbor node of the node $B, C$ and $D$, but the distance between the node $A$ and $E$ is more than onehop communication range, it needs two-hop communication. In order to calculate the distance between the node $A$ and $E$, take the node $B$ as the origin, take the line $B C$ as $x$ axis, and take the plane defined by node $B, C$ and $D$ as the $x y$-plane to build a three dimensional coordinate system. Because the node $A$ is the neighbor node of the node $B, C$ and $D$, the length of $A B, A C$ and $A D$ can be calculated by utilizing RSSI ranging method. In a similar way, the node $B, C$, and $D$ are the neighbor nodes with each other, and the node $E$ is the neighbor node of the node $B, C$, and $D$, the length of $B C, B D$ and $C D$ as well as $B E, C E$ and $D E$ can be also calculated by utilizing RSSI ranging. In the coordinate system in Figure 1, suppose that the coordinates of the node $B$ is $(0,0,0)$, the coordinates of the node $C$ is $(B C, 0,0)$, and the coordinates of the node $D$ is $\left(x_{d}, y_{d}, 0\right)$, then $x_{d}$ and $y_{d}$ satisfies the following equations.

$$
\left\{\begin{array}{c}
\left(x_{d}-B C\right)^{2}+y_{d}^{2}=C D^{2} \\
x_{d}^{2}+y_{d}^{2}=B D^{2}
\end{array}\right.
$$

By solving the equations, the value of $x_{d}$ and $y_{d}$ can be obtained as follows.

$$
\left\{\begin{array}{c}
x_{d}=\frac{B C^{2}+B D^{2}-C D^{2}}{2 B C} \\
y_{d}= \pm \frac{\sqrt{(B C+B D+C D)(B C+B D-C D)(B C-B D+C D)(B D-B C+C D)}}{2 B C}
\end{array}\right.
$$

Where the positive value or negative value of $y_{d}$ only changes the position of the node $D$ in the three dimensional coordinate system, not changing the length of $A E$. In this way, the coordinates of the node $B, C$ and $D$ can be calculated. Suppose the coordinates of the node $A$ in Figure 1 is $\left(x_{a}, y_{a}, z_{a}\right)\left(z_{a}>0\right)$, the equations (4) can be gotten using the distance equation between nodes.

$$
\left\{\begin{array}{c}
x_{a}^{2}+y_{a}^{2}+z_{a}^{2}=A B^{2} \\
\left(x_{a}-B C\right)^{2}+y_{a}^{2}+z_{a}^{2}=A C^{2} \\
\left(x_{a}-x_{d}\right)^{2}+\left(y_{a}-y_{d}\right)^{2}+z_{a}^{2}=A D^{2}
\end{array}\right.
$$


Because the length of $A B, A C, B C$ and $A D$ has been known, the value of $x_{a}, y_{a}$, and $z_{a}$ can be solved as follows.

$$
\left\{\begin{array}{c}
x_{a}=\frac{A B^{2}-A C^{2}+B C^{2}}{2 B C} \\
y_{a}=\frac{A B^{2}-A D^{2}-2 x_{a} x_{d}+B D^{2}}{2 y_{d}} \\
z_{a}=\sqrt{A B^{2}-\frac{\left(A B^{2}-A C^{2}+B C^{2}\right)^{2}}{4 B C^{2}}-\frac{\left(A B^{2}-A D^{2}-2 x_{a} x_{d}+B D^{2}\right)^{2}}{4 y_{d}^{2}}}
\end{array}\right.
$$

In a similar way, the coordinates of the node $E\left(x_{e}, y_{e}, z_{e}\right)$ can be calculated, and the length of $A E$ can be computed by the equation (6).

$$
A E=\sqrt{\left(x_{a}-x_{e}\right)^{2}+\left(y_{a}-y_{e}\right)^{2}+\left(z_{a}-z_{e}\right)^{2}}
$$

When calculating the length of $A E$ from geometry, there may be two cases, that is, $A E$ or $A E^{\prime}$, as shown in Figure 2. It is allowed to use the following method to solve this problem.

Node $A$ and node $E$ are not neighbor nodes, but can communicate with two-hop, thus, the following constraint need to satisfy, i.e., $R<A E<2 R$, where $R$ is the communication radius of the node. If the two values of $A E$ are satisfied the constraint condition through the calculation, the further judgments are carried out. Select a node among the neighbor nodes of the node $E$, and this node cannot be the node $B, C$, or $D$, and use this node to replace any one of the three nodes $B, C$ and $D$. In this way, other two values of $A E$ can also be obtained. In these four values, select two values that are close to each other, and take the average value of the two values as the length of $A E$. In the three dimensional environment, after each node uses the same method as the node $E$ to calculate the distance from four anchor nodes to itself, it is allowed to use the maximum likelihood estimation method to calculate its own coordinates.

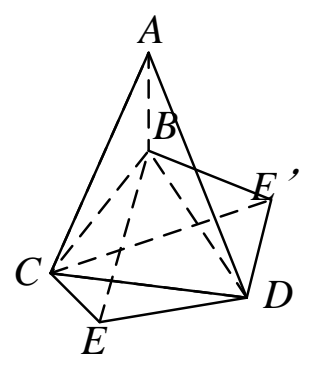

Figure 2. The Two Cases of the Line Segment $A E$ in Three Dimensions

\section{The Procedure of the Localization Algorithm}

Before describing the localization algorithm, we make some explanations. All the wireless sensor nodes are disposed randomly in a certain three dimensional space. All the nodes can be one-hop ranging, and the ranging error obeys the Gaussian distribution. All the nodes have the same communication capability and the same communication radius. The nodes can directly communicate with each other within the range of communication. Each node has a unique identity in the whole network, and has the same data processing capability. The anchor nodes are equipped with the global positioning system. The communication between the nodes is in the line-of-sight case, because in the non-line-ofsight case the ranging error between the nodes is very uncertain, and the node ranging is beyond the scope of this paper. 
Next, we give the detailed procedure of the node localization algorithm.

Step 1: All the nodes are deployed randomly in the region within a certain range. Each anchor node obtains its own absolute coordinates through the global positioning system, and sets the node type attribute as 'anchor node'. Every non-anchor node sets the node type attribute as 'unknown node', and sets the coordinates as $(-1.0,-1.0,-1.0)$.

Step 2: All the nodes broadcast the one-hop hello data packet, and the contents include the node ID, the node type, and the node coordinates.

Step 3: After receiving the hello data packet, each node utilizes the RSSI ranging method to calculate the distance between itself and other nodes. At the same time, if a node receives the hello data packet from an anchor node or a reference node, it stores the information of this data packet and the distance between two nodes in the anchor node list, otherwise it stores the received information and the distance in the neighbor list. For an unknown node, when it receives the hello data packet for the first time, it starts a timer. The timer examines the number of the nodes stored in the anchor node list at regular intervals. If the number is greater than or equal to 4, the unknown node can use the equation (1) to calculate its coordinates, and sets the node type attribute as 'reference node'.

Step 4: The anchor nodes and reference nodes broadcast the two-hop localization data packet, and the contents include the node ID, the node type, and the broadcasting hop count.

Step 5: After a node receives the broadcasting data packet, the corresponding operation is carried out according to the following two cases.

Case 1: If the broadcasting hop count is 2 , it indicates that the location of this node is similar to the node $B, C$ or $D$ in Figure 1 . Then, the node adds its own information in this localization data packet including the node ID and the neighbor list, and then it broadcasts this data packet after reducing the broadcasting hop count by 1 .

Case 2: If the broadcasting hop count is 1, there are two cases as follows.

Case a: If the source node of the broadcast packet is its neighbor node, the path taken by this broadcast packet is equivalent to the following path, i.e., starting from the node $A$, through one of the three nodes $B, C$, and $D$, and reaching one of the two residual nodes. Therefore, the location of the node that receives the broadcast packet is similar to the node $B, C$ or $D$. In this case, the node does not need to do any operation on this broadcast packet.

Case b: If the source node of the broadcast packet is not its neighbor node, the location of the node that receives the broadcast packet is similar to the node $E$ in Figure 1. The node needs to judge whether the constraint can be satisfied, i.e., $R<A E<2 R$. If the constraint condition is satisfied, the node calculates the distance between itself and the source node, and stores the information of the source node and the calculated distance in the anchor node list. If the constraint condition is not satisfied, this node will wait for another broadcasting data packet of the same source node.

Step 6: If the coordinates of all the nodes have been known, the localization algorithm runs over. Otherwise, the newly upgraded reference nodes repeat the Step 4.

\section{Simulation and Evaluation}

In this section, we present the results of simulating the proposed node localization algorithm. We select OPNET 17.5 as the platform and use the three-layer modeling mechanism to simulate the performance of our algorithm compared with the traditional quadrilateral localization algorithm.

\subsection{The Network Model}

The simulation scenario is set to a square monitoring region that the side length is 2000 m. There are 200 wireless sensor nodes are deployed randomly, as shown in Figure 3, 
where the ID of the nodes is $1,2, \ldots$, and 200 , respectively. The functions of all the nodes are the same and they are determined by the node model. In addition, there is a special node, i.e., node_0. The node_0 is a receiver groups that can set up the communication radius, channel, bandwidth and so on of all the nodes to make all the nodes have the same communication capability. In each experiment, different number of the anchor nodes is set according to the different proportion of the anchor nodes. The localization algorithm does not consider the non-line-of-sight case, so no obstacle is placed between the nodes in the network model.

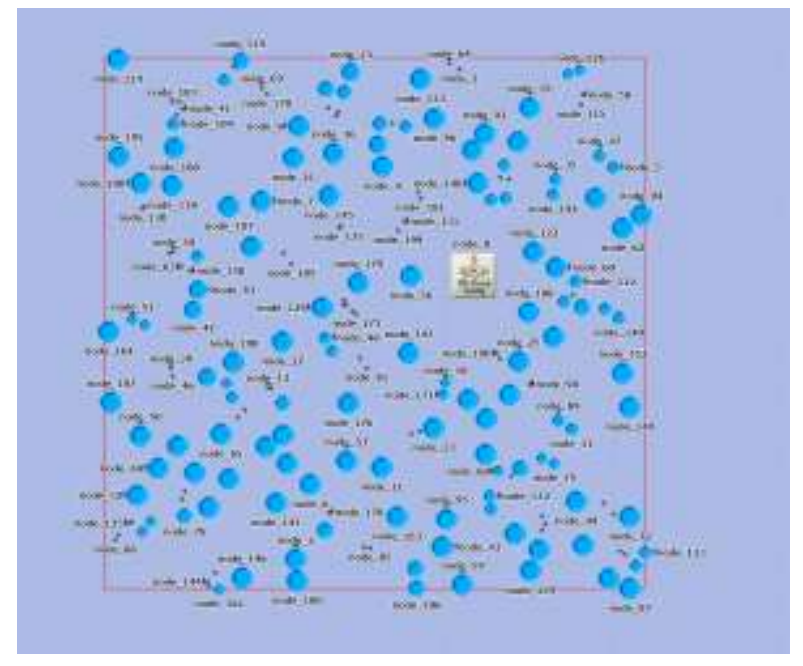

Figure 3. The Network Model

\subsection{The Node Model}

The node model is shown in Figure 4. The node model consists of four modules, i.e., the queue module (q_0), wireless receiver module (rr_0), wireless transmitter module (rt_0), and antenna module (a_0). Among them, the queue module is the main module of implementing the localization algorithm, and the function is determined by the corresponding process model. The wireless receiver module and wireless transmitter module define the standard physical layer model, and the node model receives and transmits the data packet through the antenna module.

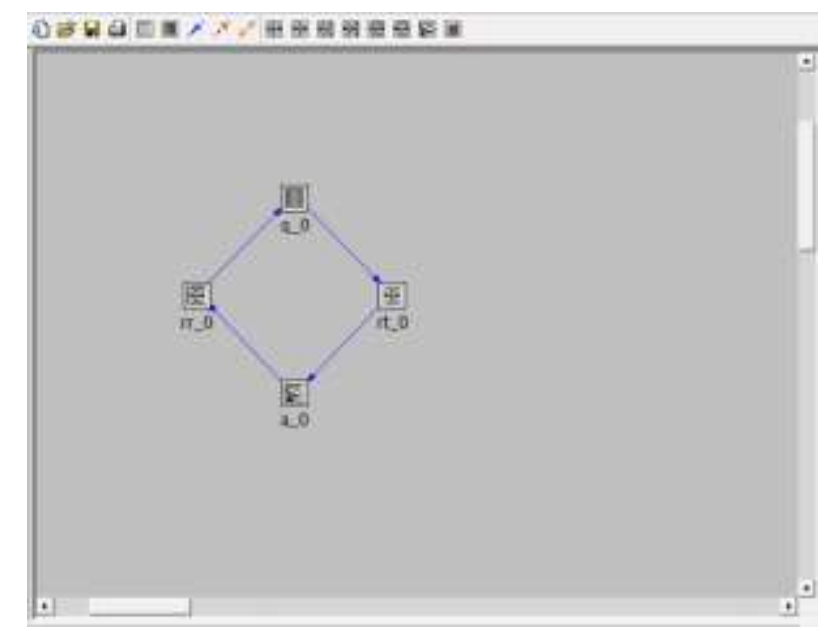

Figure 4. The Node Model 


\subsection{The Process Model}

The process model is the key part of the implementation of the localization algorithm. The process model is shown in Figure 5. There are two states in the process model including initial state (st_0) and waiting state (st_1). The initial state initializes the properties of the nodes including the node type and the node coordinates. The waiting state waits for the arrival of all kinds of interrupts and performs the corresponding interrupt program, and the interrupts include the self interrupts and flow interrupts. In Figure 5, after the state of st_0 finishes the initialization, it will switch to the state of st_1 automatically. Next, the state of st_1 will produce a self interrupt SELF_INTRPT and the process model will call the function of send_hello() automatically. This function makes the node broadcast the hello data packet to the neighbor nodes. PACKETARR is the flow interrupt, and when the state of st_1 receives this interrupt, it indicates that the node has received the data packet, and the process model will call the function of recv() automatically. The function of $\operatorname{recv}()$ will implement the corresponding procedures according to the type of the data packet. If it is a hello data packet, it will store the node information; if it is a localization data packet, it will perform the localization algorithm.

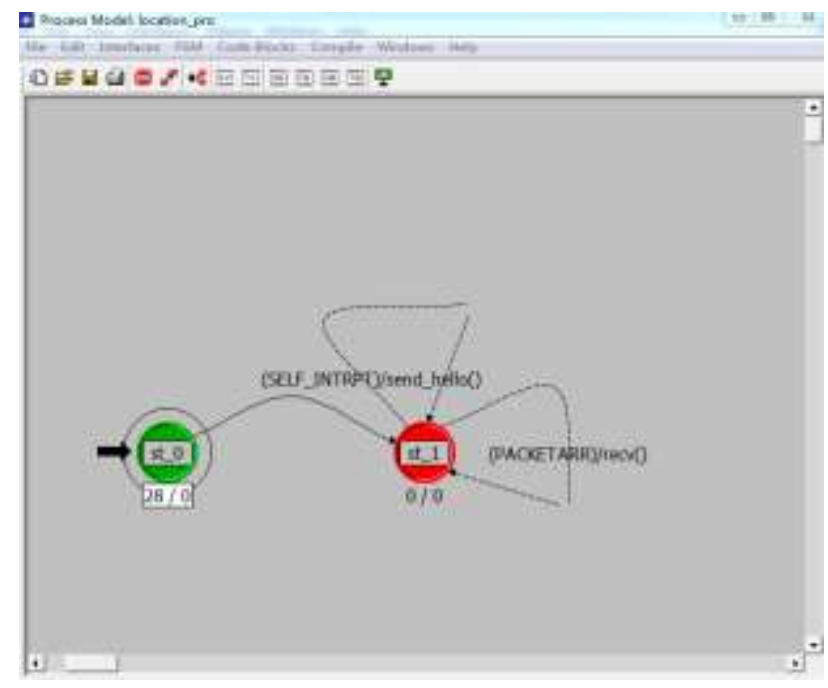

Figure 5. The Process Model

The variables used in the process model are shown in Figure 6, where $x, y$ and $z$ is the actual three dimensional coordinates of the node, and $c a l \_x, c a l \_y$ and $c a l \_z$ is the coordinates obtained by the calculation of the localization algorithm. PregT_List is a special data structure in OPNET, which is similar to the list structure. The variables $n b \_l i s t$ and anchor_node is the PregT_List data type, which are used for storing the related information of the neighbor nodes and the localization data packet, respectively. 


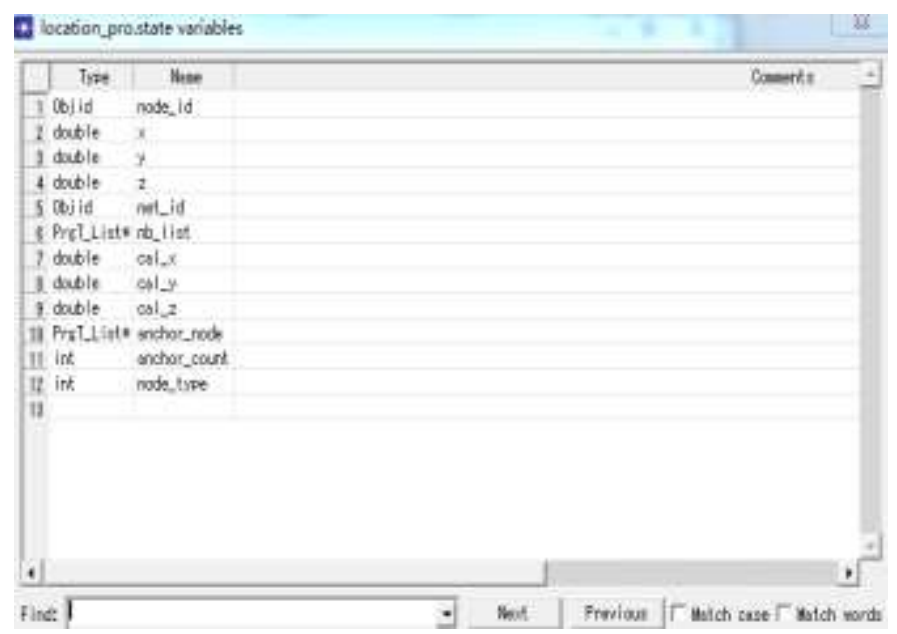

Figure 6. The Variables in the Process Model

The data packets used in localization algorithm is shown in Figure 7. The field 'type' indicates the type of the data packet including the hello data packet and the localization data packet. The field 'ttl' denotes the maximum value of the hop count of the data packet, and when the value of 'ttl' is equal to 0 , the node directly abandons the data packet. The field 'source' and 'relay' are used to store the information of the source node and the relay node, respectively. The field 'distance' is used to store the distance between the source node and the relay node. The field 'neighbors' is a variable field used to store the information of the neighbor nodes.

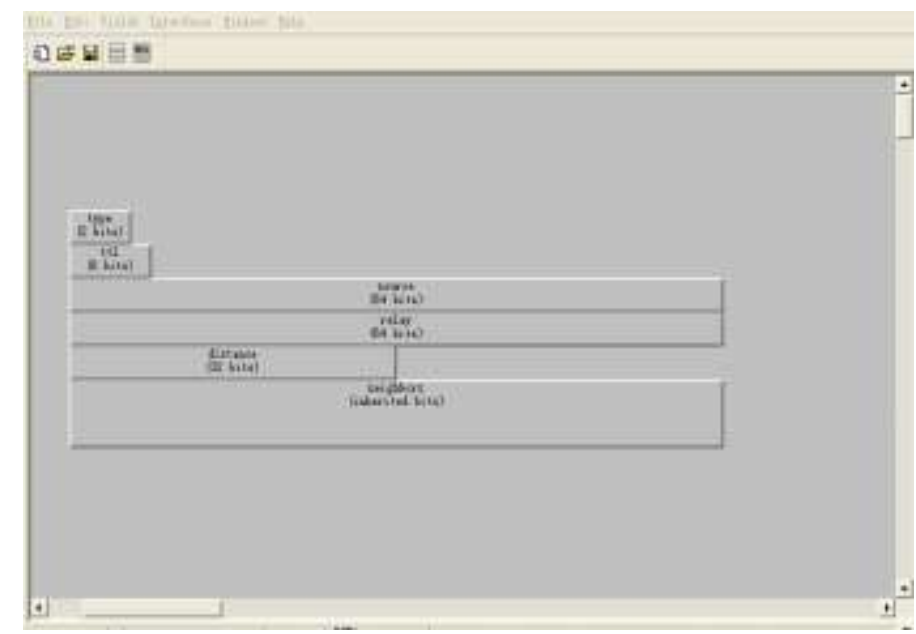

Figure 7. The Data Packets

\subsection{Definition of the Simulation Indicator}

In this paper, we select two simulation indicators to evaluate the performance of the proposed algorithm, i.e., localization precision and localization coverage rate, which have a certain representation for the evaluation of the localization algorithm.

The localization precision is the most important indicator to evaluate the localization algorithm. It is allowed to consider the localization precision in two aspects: on the one hand, it is the localization precision of each node, and on the other hand, it is the average localization precision of the whole network. Because the localization precision of every node may be higher or lower, it is not suitable for evaluating the localization algorithm. Therefore, we use the average localization precision of the whole network. The localization precision is represented by the node relative localization error, and the 
smaller the relative localization error is, the higher the localization precision is. The average relative localization error $\bar{e}$ of the whole network is defined by the equation (7).

$$
\bar{e}=\frac{\sum e_{i}}{n R}
$$

Where $e_{i}=\sqrt{\left(x_{i}+x_{i}^{\prime}\right)^{2}+\left(y_{i}+y_{i}^{\prime}\right)^{2}+\left(z_{i}+z_{i}^{\prime}\right)^{2}}$ denotes the localization error of the node $i,\left(x_{i}, y_{i}, z_{i}\right)$ is the calculated coordinates of the node $i,\left(x_{i}^{\prime}, y_{i}^{\prime}, z_{i}^{\prime}\right)$ is the real coordinates of the node $i, n$ is the number of the nodes in the network, and $R$ is the communication radius of the node.

The localization coverage rate refers to the ratio between the number of the nodes positioned by the localization algorithm and the total number of the unknown nodes. The localization coverage rate $c$ is defined by the equation (8).

$$
c=\frac{\text { located }}{\text { sum }}
$$

Where located denotes the number of the nodes positioned by the localization algorithm and sum denotes the number of the nodes other than the anchor nodes in the network.

As can be seen from the equation (8) that under the condition of guaranteeing certain localization precision, the higher the localization coverage rate is, the better the performance of the algorithm is.

\subsection{The Simulation Results}

Next, we present the simulation results of our localization algorithm compared with classical quadrilateral localization algorithm in terms of the localization precision and the localization coverage rate.

\subsubsection{The Localization Precision}

The communication radius of the node is set to $300 \mathrm{~m}$, and the simulation results of the relative localization error are shown in Figure 8.

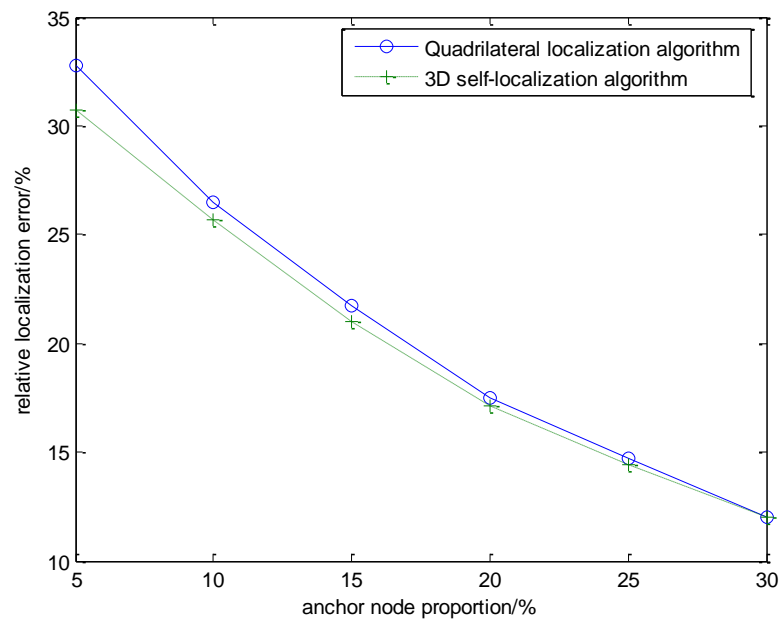

Figure 8. The Relative Localization Error

As can be seen from Figure 8, the relative localization error of quadrilateral localization algorithm is larger. When the density of the anchor nodes increases, the localization accuracy of the two algorithms is getting closer. The reason is that when the 
density of the anchor nodes is large enough, the proposed localization algorithm is similar to the quadrilateral localization algorithm.

\subsubsection{The Localization Coverage Rate}

The simulation results of the localization coverage rate of two algorithms are given in Figure 9, Figure 10 and Figure 11 when the communication radius $R$ is set to $200 m, 300$ $m$ and $400 m$, respectively.

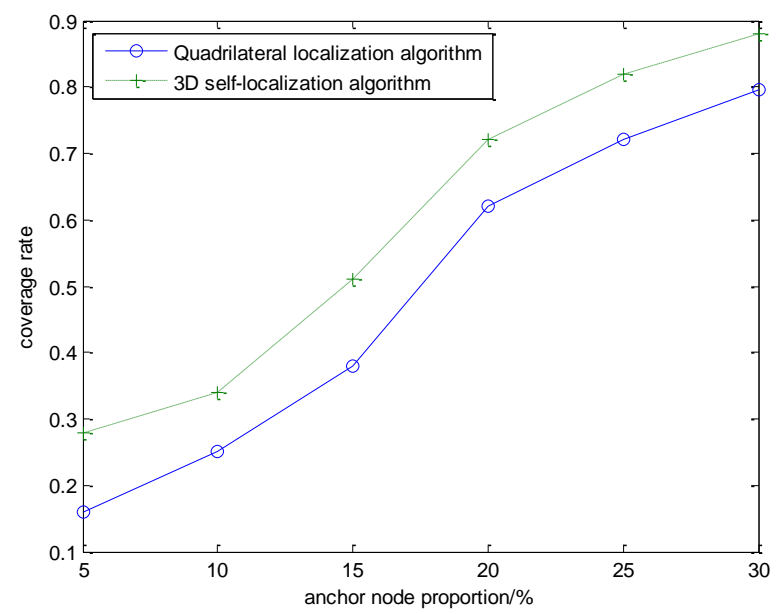

Figure 9. The Localization Coverage Rate $(R=200 \mathrm{~m})$

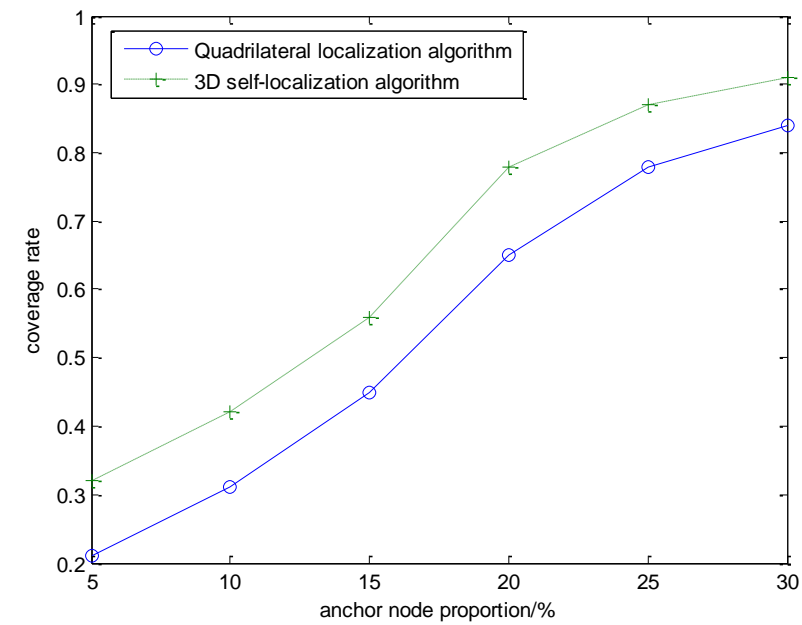

Figure 10. The Localization Coverage Rate $(R=300 \mathrm{~m})$ 


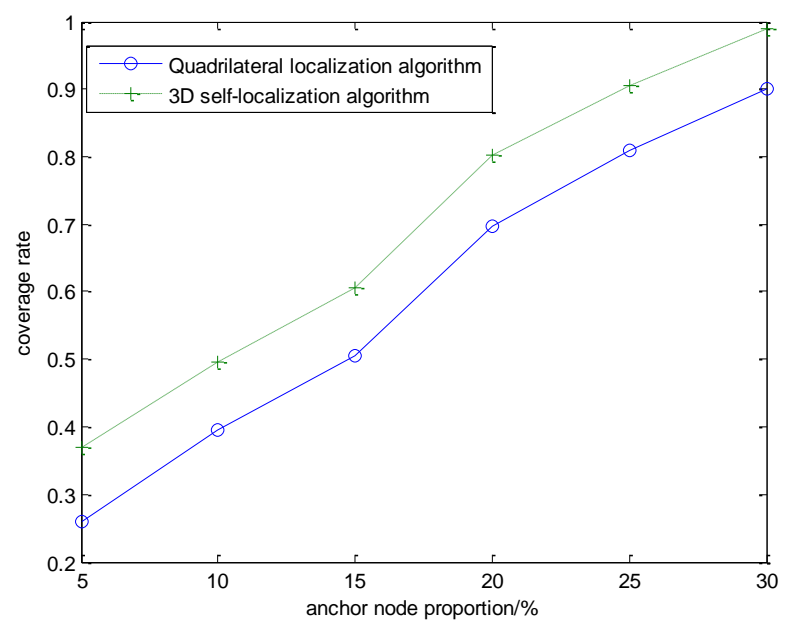

Figure 11. The Localization Coverage Rate $(R=400 \mathrm{~m})$

As can be seen from Figure 9, Figure 10 and Figure 11, with the same communication radius and the density of the anchor nodes, the localization coverage rate of our algorithm is obviously higher than that of the quadrilateral localization algorithm, and the reason is that the proposed localization algorithm can effectively increase the proportion of the nodes positioned by the localization algorithm in the whole network.

\section{Conclusion}

The wireless sensor network is an advanced research field involving multiple subjects and the integrated knowledge, which has broad application prospects. As the core technique of wireless sensor network, the node self-localization technology is one of the research focuses at present. In recent decade, some results of study on the node localization have been obtained. However, most of these results are based on the two dimensional environment, which is difficult to meet the requirements of the deployment environment of wireless sensor network. In addition, the existing localization algorithms are based on the mechanism of multi-hop ranging, and with the increase of the hop counts, the accumulation of the localization error will increase gradually, which is unable to guarantee the localization precision. Aiming at these problems, a range-based three dimensional self-localization algorithm is presented in this paper. Under the premise of not adding new hardware equipment, the relative coordinate system is established by the position relation of the nodes. On this basis, according to the spatial geometry relation, the two-hop ranging method is introduced in the three dimensions, and further, the procedure of the node self-localization algorithm is given. Finally, the algorithm is simulated based on OPNET, and the network model, the node model and the process model is established, respectively. The simulation results indicate that comparing with classical quadrilateral localization algorithm, the proposed localization algorithm can effectively reduce the localization error and achieve higher localization coverage rate.

\section{References}

[1] V. Gungor and G. Hancke, "Industrial Wireless Sensor Networks: Challenges, Design Principles, and Technical Approaches", IEEE Transactions on Industrial Electronics, vol. 56, no. 10, (2009), pp. 42584265 .

[2] S. Schlupkothen, G. Dartmann, and G. Ascheid, "Exploration with Massive Sensor Swarms", Proceedings of IEEE Global Conf. on Signal Inf. Process. (GlobalSIP), (2013) December, pp. 149-152.

[3] J. Aspnes, T. Eren, D. K. Goldenberg, "A Theory of Network Localization”, IEEE Transactions on Mobile Computing, vol. 5, no. 12, (2006), pp. 1-16. 
[4] Z. Yang and Y. Liu, "Understanding Node Localizability of Wireless Ad Hoc and Sensor Networks", IEEE Trans. Mobile Computing, vol. 11, no. 8, (2012), pp. 1249-1260.

[5] M. Rudafshani and S. Datta, "Localization in Wireless Sensor Networks", Proceedings of the 6th International Symposium on Information Processing in Sensor Networks, (2007), pp. 51-60.

[6] L. Cheng, C. Wu, Y. Zhang, "A Survey of Localization in Wireless Sensor Network", International Journal of Distributed Sensor Networks, vol. 2012, no. 1, (2012), pp. 523-534.

[7] J. Kuriakose, V. Amruth, N.S. Nandhini, "A Survey on Localization of Wireless Sensor Nodes", Proceedings of 2014 International Conference on Information Communication and Embedded Systems, (2014), pp. 1-6.

[8] H. Wang, Z. Gao and Y. Guo, "A Survey of Range-Based Localization Algorithms for Cognitive Radio Networks", Proceedings of the 2nd International Conference on Consumer Electronics, Communications and Networks, (2012), pp. 844-847.

[9] L. Heng and G. Gao, "Accuracy of Range-Based Localization Schemes in Random Sensor Networks: A Lower Bound Analysis", Proceedings of IEEE/RSJ Int. Conf. Intell. Robots Syst. (IROS), (2013), pp. 907-912.

[10] P. Tarrio, A.M. Bernardos and J. R. Casar, "Weighted Least Squares Techniques for Improved Received Signal Strength Based Localization", Sensors, vol. 11, no. 9, (2011), 8569-8592.

[11] T. He, C. Huang and B. Blum, "Range-Free Localization Schemes for Large Scale Sensor Networks", Proceedings of the 9th Annual International Conference on Mobile Computing and Networking ACM, (2003), pp. 81-95.

[12] D. Niculescu and B. Nath, "Ad Hoc Positioning System (APS)", Proceedings of IEEE Global Telecommunications Conference, (2001), pp. 2926-2931.

[13] D. Niculescu and B. Nath, "DV Based Positioning in Ad Hoc Networks", Telecommunication Systems, vol. 22, no. 1, (2003), pp. 267- 280.

[14] S. Tomic, I. Mezei, "Improvements of DV-Hop Localization Algorithm for Wireless Sensor Networks", Telecommunication Systems, vol. 61, no. 1, (2016), pp. 93-106.

[15] U.A. Khan, S. Kar, and J. M. F. Moura, "Distributed Sensor Localization in Random Environments Using Minimal Number of Anchor Nodes", IEEE Transactions on Signal Processing, vol. 57, no. 5, (2009), pp. 2000-2016.

[16] J. Sheu, W. Hu, and J. Lin, "Distributed localization scheme for mobile sensor networks", IEEE Transactions on Mobile Computing, vol. 9, no. 4, (2010), pp. 516-526.

[17] T. Kim, M. Shon and M. Kim, "Anchor-node-based distributed localization with error correction in wireless sensor networks", International Journal of Distributed Sensor Networks, vol. 36, no. 1, (2012), pp. 1-14.

[18] S. Pandey and S. Varma, "A Range Based Localization System in Multihop Wireless Sensor Networks: A Distributed Cooperative Approach", Wireless Personal Communications, vol. 86, no. 2, (2016), pp. 615-634

\section{Authors}

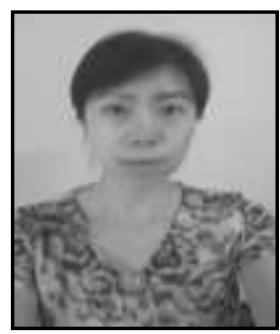

Yanhui Lv, she received the master's degree in computer application technology from Shenyang Ligong University in 2005, and the Ph.D. degree in computer application technology from Northeastern University in 2010. Now, she is a professor working in the College of Information Science and Engineering, Shenyang Ligong University. Her current research interests include wireless sensor network and system simulation.

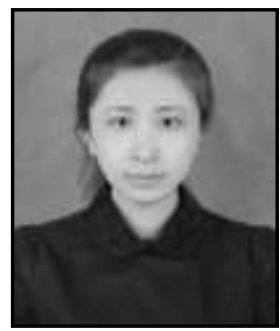

Sitong Meng, she received the B.E. degree in computer science and technology from Shenyang Ligong University in 2015. Currently, she is a master degree candidate for computer application technology at Shenyang Ligong University. Her research interests include wireless network and control system. 


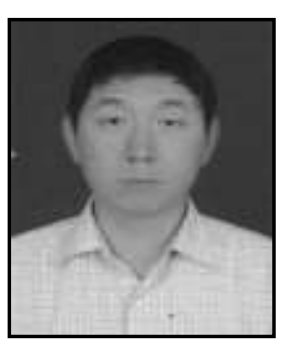

Deyu Zhang, he received the Ph.D. degree in computer science from Nanjing University of Science and Technology in 2005. Currently, he is a professor working in the College of Information Science and Engineering, Shenyang Ligong University. His research interests include wireless network, artificial intelligence and embedded system.

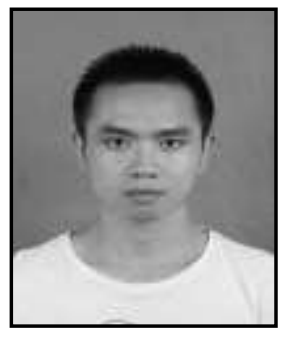

Yunfei Huang, he received the B.E. degree in computer science and technology from Shenyang Ligong University in 2012, and the master's degree in computer system structure from Shenyang Ligong University in 2015. His research interests include wireless sensor network and embedded system. 
International Journal of Multimedia and Ubiquitous Engineering

Vol. 10, No. 6 (2017) 